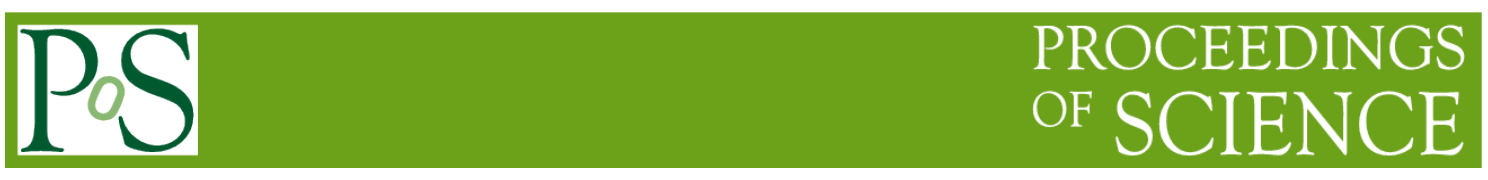

\title{
Advancements of THGEM development in IHEP, China
}

\section{Yuguang Xie ${ }^{1}$ Junguang Lv Tao Hu}

Institute of High Energy Physics, Chinese Acadmy of Science

State Key Laboratory of Particle Detection and Electronics

19B, YuQuan Rd, Beijing, China

E-mail: ygxie@ihep.ac.cn

E-mail: Iujg@ihep.ac.cn

E-mail: huteihep.ac.cn

As one of the promising Micro-pattern Gaseous Detectors (MPGDs), Thick Gaseous Electron Multiplier (THGEM) is attractive due to its high gain, robust and low cost. In order to promote its applications, continuous efforts had been making to improve the performances and production technologies of THGEMs. The advancements in both development and applications are presented, including high spatial resolution, new substrates, new structures, big area, neutron detection, CCD-based optical readout, and Digital Hadron Calorimeter (DHCAL).

The 39th International Conference on High Energy Physics (ICHEP2018)

4-11 July, 2018

Seoul, Korea 
Introduction

Thick Gaseous Electron Multiplier (THGEM) [1] is one of the promising Micropattern Gaseous Detectors and suitable for Digital Hadron Calorimeter (DHCAL), TPC tracker readout, muon detector, single photon detector, neutron imaging detector, and so on. The attractive advantages of THGEM are high gain, robust and low cost. The THGEM large-scale applications are limited by the big-area mass production, moderate spatial resolution and high gain stability. In recent years, we have made continuous efforts to improve THGEM performances, develop new types of THGEMs, and try new production technologies around its applications [2-3]. New substrates, new structures, high spatial resolution and big area are the four main directions of THGEM technologies and applications.

\section{THGEM development}

\subsection{High spatial resolution}

High spatial resolution is always a key index for most positioning detectors. As for THGEM or GEM type of MPGDs, the intrinsic spatial resolution is determined by the hole pitch, which is normally about $140 \mu \mathrm{m}$ and $500 \mu \mathrm{m}$ for GEM and THGEM, and the spatial resolution reaches $\leq 47 \mu \mathrm{m}$ and $\leq 167 \mu \mathrm{m}$ respectively ( $\leq$ pitch $/ \sqrt{ } 12)$. An expected resolution is at $100 \mu \mathrm{m}$ for tracking or imaging applications. So THGEMs with smaller hole pitch and hole diameter were developed, for example, 150- $\mu \mathrm{m}$ hole diameter and 400- $\mu \mathrm{m}$ hole pitch, and the spatial resolution measured is about $75 \mu \mathrm{m}$ [4]. By laser etching, a new technology for THGEM production, the hole diameter and pitch can reach $100 \mu \mathrm{m}$ and $300 \mu \mathrm{m}$ respectively, and so the intrinsic spatial resolution could reach around $56 \mu \mathrm{m}$ or better.
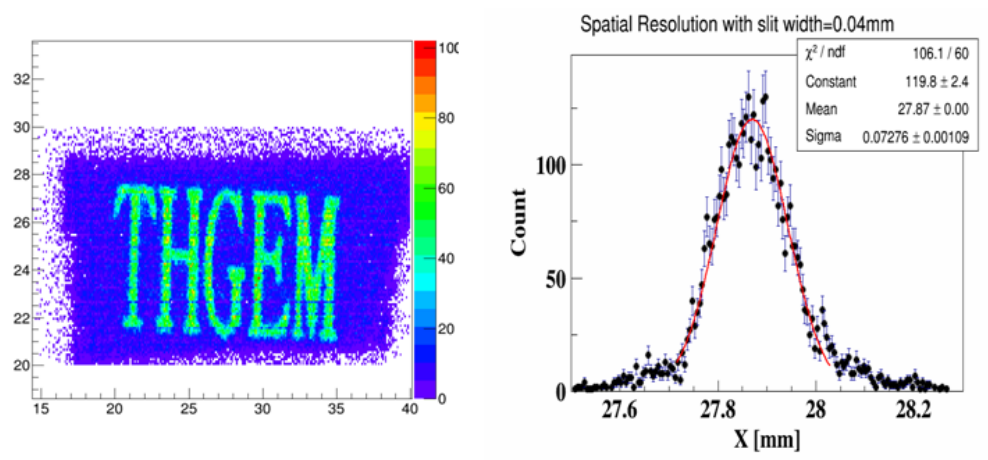

Fig. 1 X-ray imaging with high spatial resolution THGEM

\subsection{New substrates}

Not like the standard GEM with only one substrate and one size, THGEM could be made by many new substrates besides FR-4, such as Kapton (PI), PTFE, ceramic, and so on. For thermal neutron detection, the ceramic THGEM has lower neutron scattering and absorption [3]. For low background experiments, substrates with low radioactivity and low out-gassing are required. The candidates include PTFE, Kapton and new FR-4. The new FR-4 substrate is developed aiming at low cost, high gain, long-life and big-area. All of these kinds of substrates had been applied to THGEM 
production, and the performances are equal to or better than that of normal FR-4 THGEM.

\subsection{New structures}

Some new structures were explored based on the existing MPGDs to overcome insuficent performance and realize more compact detectors. For THGEM, a promissing new structure is the multi-layer THGEM (M-THGEM) [5]. M-THGEM combines two or three layers of normal THGEMs into one film by using the multilayer PCB technology. M-THGEM can reach equal high gain as the trandictional two layers of cascade THGEMs, with thinner structure by removing the transfer gap. More importantly, M-THGEM saves about one half production cost due to form two layers of avalanching holes by one time drilling. That means the performance of one M-THGEM is equal to that of two layers of THGEMs, but the production time is only half.
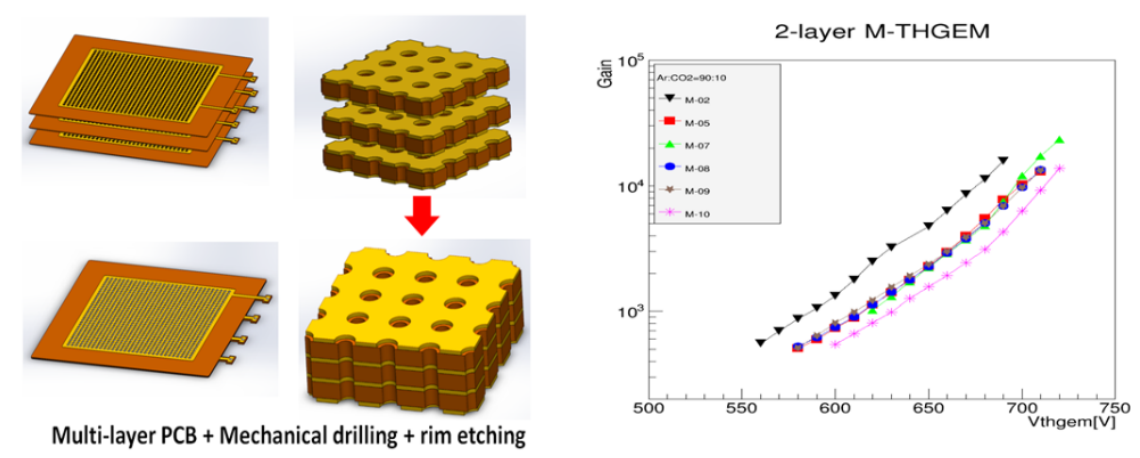

Fig. 2 The structure (left) and gain (right) of M-THGEM

\subsection{Big area}

Big area and mass production are the key issures for THGEM application on particle physics experiments. A big size of THGEM, e.g. $1000 \times 500 \mathrm{~mm}^{2}$, were produced to verify the potential of mechanical drilling technology. However, the time consumption is too high and the yield is low. By using the laser etching, the time of hole forming is reduced greatly, only about $1 / 10$ of mechanical drilling. The yield is still unsatisfactory and need improving.

\section{THGEM applications}

\subsection{Neutron detection}

THGEM is thought as one more suitable MPGD for neutron detection due to its moderate spatial resolution and robust. A new themal neutron detection structure was proposed and tested as showed in Fig. 3 [6]. The ideal is to seperate the neutron conversion area and the electron avalanch area by coating the Boron on meshes instead of THGEM or GEM films, and only one layer of THGEM is used for signal amplification. The advantages of this structure are easy to coat Boron on multi-layer meshes with different meterials and structures, without impacts on THGEMs or GEMs themselves, and both meshes and THGEMs are replaced. 

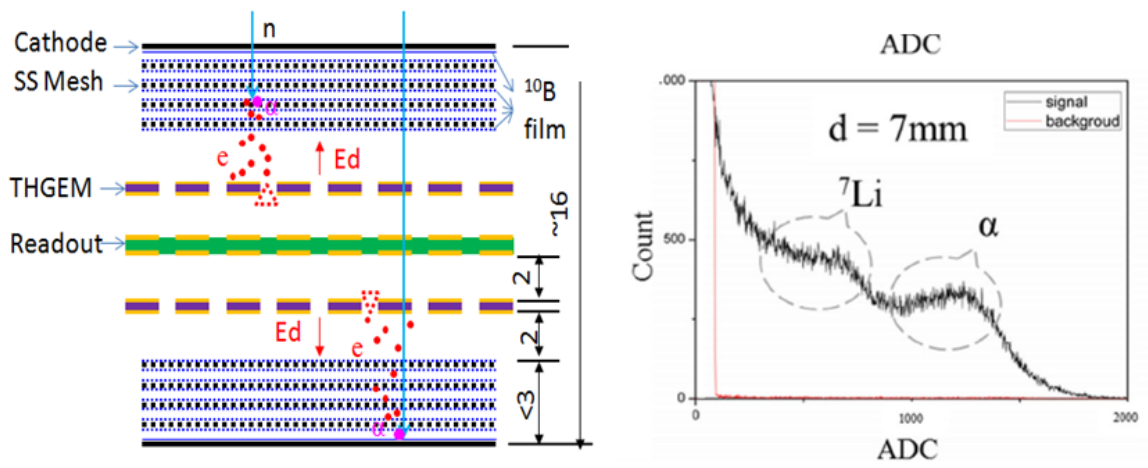

Fig. 3 A new THGEM-based neutron detector (left) and the test result (right)

\subsection{CCD-based optical readout}

It is interesting to realize the imaging by using the CCD (not ICCD) camera with a THGEM detector instead of the tradictional multi-channel analog readout. The visable light emitted during the avalaching makes this possible if the light yield is enough, and this requres high-gain THGEM and high-light-yield gas mixture, such as CF4. Fig. 4 shows the imaging and spatial resoltuion measured results of the $60 \times 60 \mathrm{~mm}^{2}$ THGEM detector by X-ray[7], more large sensitive area detector with higher spatial resolution is being developped.
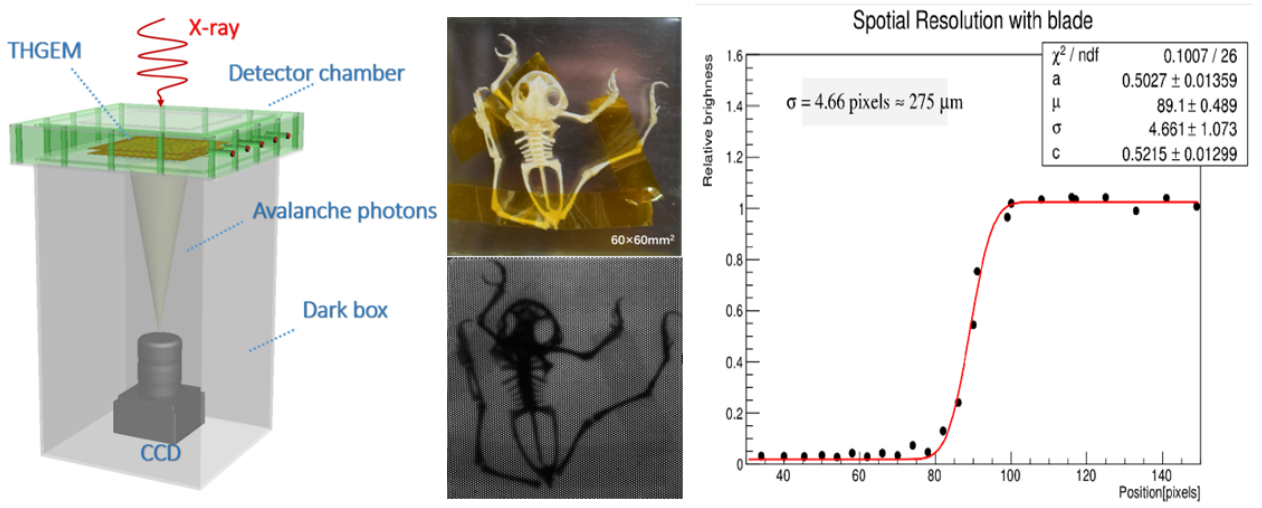

Fig. 4 The preliminary imaging result by CCD readout

\subsection{DHCAL and muon detector}

As mentioned above, the M-THGEM is a good candidate applied to DHCAL and muon detector. Both DHCAL and muon detector require high gain, robust and compact. The total thickness and cost can be reduced and the assembly will be more convenient if M-THGEM was chosed instead of normal THGEM. A DHCAL protoype made of M-THGEM and ASIC readout is under study.

\section{References}

[1]R. Chechik et al., Nuclear Instruments and Methods in Physics Research A 535 (2004) 303

[2]Y.G Xie et.al, Nuclear Instruments and Methods in Physics Research A 729 (2013) 809-815

[3]X.Zhang,S. Niu, Y.Xie* et.al, 2015 JINST 10 P10043 
[4]Hang Zhao , et.al., Radiation Detection Technology and Methods, https://doi.org/10.1007/s41605017-0009-z

[5]M. Cortesi et al., arXiv:1606.07314 [physics.ins-det]

[6]Li, G., et al., Radiation Detection Technology and Methods, https://doi.org/10.1007/s41605-0180055-1

[7] Peng, ZY, et al., Radiation Detection Technology and Methods, https://doi.org/10.1007/s41605018-0058-y 